\title{
Can the surface electrocardiogram be used to predict myocardial viability?
}

\author{
A Al-Mohammad, M Y Norton, I R Mahy, J C Patel, A E Welch, P Mikecz, S Walton
}

\begin{abstract}
Objective-To investigate whether QRS morphology on the surface ECG can be used to predict myocardial viability.

Design-ECGs of 58 patients with left ventricular impairment undergoing positron emission tomography (PET) were studied. ${ }^{13} \mathrm{~N}$-Ammonia $\left(\mathrm{NH}_{3}\right)$ and ${ }^{18} \mathrm{~F}$ fluorodeoxyglucose (FDG) were the perfusion and the metabolic markers, respectively. The myocardium is scarred when the uptake of both markers is reduced (matched defect). Reduced $\mathrm{NH}_{3}$ uptake with persistent FDG uptake (mismatched defect) represents hibernating myocardium. First, the relation between pathological $Q$ waves and myocardial scarring was investigated. Second, the significance of $Q R$ and $Q S$ complexes in predicting hibernating myocardium was determined.
\end{abstract}

Results-As a marker of matched PET defects, $Q$ waves were specific $(79 \%)$ but not sensitive (41\%), with a $77 \%$ positive predictive accuracy and a poor $(43 \%)$ negative predictive accuracy. The mean size of the matched PET defect associated with $Q$ waves was $20 \%$ of the left ventricle. This was not significantly different from the size of the matched PET defects associated with no $Q$ waves $(18 \%)$. Among the regions associated with $Q$ waves on the ECG, there were 16 regions with $Q R$ pattern (group $A$ ) and 23 regions with QS pattern (group B). The incidence of mismatched PET defects was $19 \%$ of group $A$ and $30 \%$ of group B (NS).

Conclusions- $Q$ waves are specific but not sensitive markers of matched defects representing scarred myocardium. $Q$ waves followed by $R$ waves are not more likely to be associated with hibernating myocardium than QS complexes.

(Heart 1999;82:663-667)

Keywords: electrocardiography; myocardial viability; positron emission tomography; myocardial scarring

The PET Unit, Department of Bio-medical Physics and Bio-engineering, Aberdeen University, Foresterhill, Aberdeen, UK

M Y Norton

A E Welch

P Mikecz

Correspondence to: Dr Al-Mohammad

Accepted for publication 1 July 1999

Left ventricular impairment is the single most important predictor of mortality after myocardial infarction. ${ }^{1}$ The prognosis of patients with severe impairment is poor, but may be improved by revascularisation if the impaired myocardium is viable. ${ }^{2}$ Currently, detection of viable myocardium requires expensive diagnostic methods. Selection of patients for further investigation by simple means would be of considerable value. One possible candidate is the surface ECG.
The significance of pathological $Q$ waves on the surface ECG is controversial. Some investigators believe that $Q$ waves reflect scarred myocardium. ${ }^{34}$ Others state that the $\mathrm{Q}$ waves are dynamic rather than permanent changes after myocardial infarction. ${ }^{5} \mathrm{~A}$ third view suggests that evidence of viable myocardium can be found in many patients with pathological Q waves. ${ }^{6}$ Furthermore, it has been suggested that preservation of $\mathrm{R}$ waves after pathological Q waves is a marker for hibernating myocardium. ${ }^{2}$ This has not been tested against imaging techniques for myocardial viability.

In this study we investigated the use of the surface ECG in selecting patients for viability studies by evaluating the relation between QRS morphology and myocardial viability using positron emission tomography (PET).

\section{Methods}

PATIENTS

The ECGs of 58 patients were studied (48 male, 10 female). These were an unselected group of patients with ischaemic heart disease and impaired left ventricular contraction who were undergoing PET. The index myocardial infarct occurred at a mean of 109 weeks (range six weeks to 13 years) before imaging. This was the most recent infarction in five patients (9\%) who had multiple documented infarctions. Myocardial infarction was diagnosed retrospectively in 13 patients $(22 \%)$, when they presented with angina or left ventricular impairment. The patients' characteristics are summarised in table 1 .

THE ELECTROCARDIOGRAMS

Surface ECGs were reviewed to assess the relation of pathological $\mathrm{Q}$ waves to myocardial scarring, and the predictability of myocardial hibernation by the maintenance of $\mathrm{R}$ waves after pathological $Q$ waves. In addition, we assessed the impact of the size of the myocardial region with scarred or hibernating myocardium on the appearance of $\mathrm{Q}$ waves, or the preservation of $\mathrm{R}$ waves after $\mathrm{Q}$ waves, respectively.

Pathological Q waves were defined as wide
(> 0.04 seconds) and deep (> $4 \mathrm{~mm}$ or $>25 \%$

Table 1 Demographic characteristics and risk factors

\begin{tabular}{ll}
\hline $\begin{array}{l}\text { Number of patients } \\
\text { Age (years) (mean (SD)) }\end{array}$ & 58 \\
Male:female ratio & $62.7(8.8)$ \\
Risk factors for ischaemic heart disease & $4.3: 1$ \\
$\quad$ & \\
Hypertension & $20 / 58(34.5 \%)$ \\
Hypercholesterolaemia & $50 / 58(86.2 \%)$ \\
Diabetes mellitus & $16 / 58(27.6 \%)$ \\
Family medical history & $23 / 58(39.7 \%)$ \\
History of smoking & $43 / 58(74.1 \%)$ \\
\hline
\end{tabular}




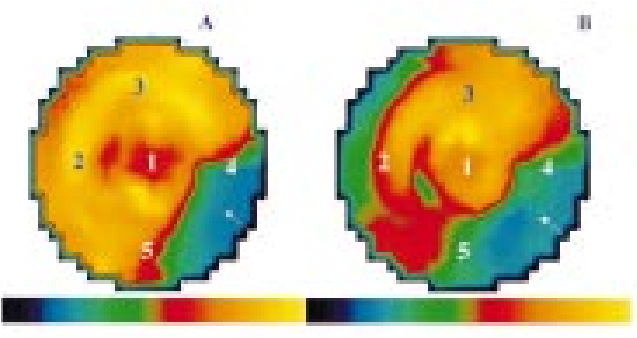

Figure 1 Two polar maps representing the left ventricle. Each is divided into five regions. The numbers represent the following left ventricular regions: 1 , the apex; 2 , the septum; 3 , the anterior wall; 4, the lateral wall; 5, the inferior wall. (A) The perfusion polar map. This shows an inferolateral defect (arrowhead). (B) The metabolic polar map. This shows a matching metabolic defect in the inferolateral region (arrowhead). Therefore, the patient has a matched inferolateral defect suggestive of myocardial scarring in that region. (The colour scale is representative of the radioactive activity in the region, with yellow being the highest count.)

of the height of the corresponding $\mathrm{R}$ wave, provided the $R$ wave was $>5 \mathrm{~mm}$ ), appearing in at least two contiguous leads.

The ECG leads were divided into two main regions:

1. The septal, anterior, and lateral walls region: leads I, aVL, and V1-6.

2. The inferior region: leads II, III and aVF. Lead aVR was excluded from analysis.

POSITRON EMISSION TOMOGRAPHY

PET was carried out after obtaining informed written consent. The locally appointed ethics committee approved the protocol.

The methods of image acquisition and analysis have been described previously. ${ }^{7}$ In brief, using a Siemens Exact 31 scanner (Siemens, Knoxville, Tennessee, USA), 31 slices were produced through the left ventricle. The reconstructed image resolution was 10 $\mathrm{mm}$. Attenuation correction was achieved by performing a transmission scan before the emission scan.

${ }^{13} \mathrm{~N}$-Ammonia and ${ }^{18} \mathrm{~F}$-fluorodeoxyglucose were used as the perfusion and metabolism markers, respectively. ${ }^{8}{ }^{18} \mathrm{~F}$-Fluorodeoxyglucose was given after an oral glucose load, and insulin was given to achieve euglycaemia in diabetic patients.

Using the filtered back projection method, images were reconstructed to generate short axis sections of the myocardium. These sections were subjected to circumference profile analysis to produce polar maps representing the left ventricle (fig 1). ${ }^{10}$

The level of highest ${ }^{13} \mathrm{~N}$-ammonia uptake was considered to represent the reference perfusion level. The glucose uptake in the area with reference perfusion level was regarded as the reference ${ }^{18} \mathrm{~F}$-fluorodeoxyglucose uptake for that patient. The perfusion and metabolism in the other areas were then compared with the perfusion and metabolism in the reference area.

Viable myocardium was defined by the presence of ${ }^{18} \mathrm{~F}$-fluorodeoxyglucose uptake. ${ }^{11}$ The viable areas with reduced ${ }^{13} \mathrm{~N}$-ammonia uptake and increased ${ }^{18} \mathrm{~F}$-fluorodeoxyglucose uptake, compared with the reference region were labelled as mismatched defects. Mismatched

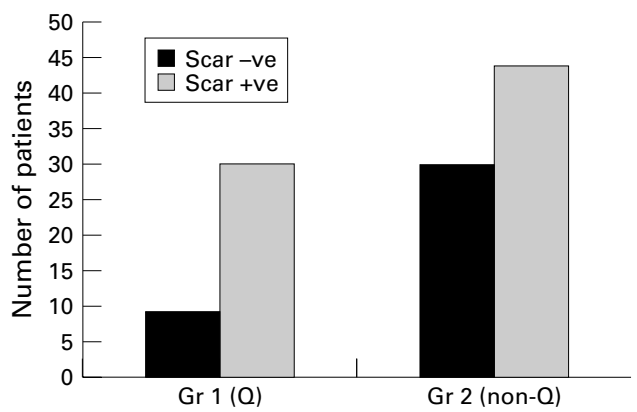

Figure 2 The importance of $Q$ wave as a marker of myocardial scarring. The bars represent the patients with (group 1) and without (group 2) pathological $Q$ waves on their surface ECG.

defects are considered to represent hibernating myocardium (although the definitive diagnosis of hibernating myocardium relies on postoperative improvement in contraction in a previously impaired region). ${ }^{12}$

Severe reduction (to a level less than $50 \%$ of the reference level) of both perfusion and metabolism activity defined a matched defect. Matched defects represent scarred myocardium. The size of a matched defect was estimated from the short axis sections of the left ventricular perfusion and metabolism images.

STATISTICAL ANALYSIS

Continuous variables are presented as mean (SD). Categorical data are presented as frequencies. The comparison between groups was performed using $\chi^{2}$ tests for categorical data. Differences with a $\mathrm{p}$ value $<5 \%$ were considered to be statistically significant. Data were analysed using Microsoft Excel Release 4.0 and SPSS for Microsoft Windows Release 6.1 software packages.

\section{Results}

Q WAVE AS A MARKER OF MYOCARDIAL SCARRING There were 116 electrocardiographic regions. These were divided into two groups according to the presence of pathological Q waves. Group $1(n=39)$ formed the regions with pathological $\mathrm{Q}$ waves, and group $2(\mathrm{n}=77)$ the regions with no pathological $Q$ waves. Matched PET defects, as evidence of scarring, were noted in 74 regions. Of these scarred regions, 30 were in group 1 and 44 in group 2 (fig 2). Therefore, the specificity of $\mathrm{Q}$ waves as markers of myocardial scarring was $79 \%$. Their sensitivity, however, was low at $41 \%$. In the cohort studied, the positive predictive value of $\mathrm{Q}$ waves for myocardial scarring was $77 \%$, and the negative predictive value was $43 \%$.

The effect of the extent of chest lead involvement by $\mathrm{Q}$ waves on the predictability of scarring was also assessed. The patients with pathological Q waves in these leads were divided into those with pathological $\mathrm{Q}$ waves in the leads V1-3 (septal), and those with pathological Q waves in the leads V1-4, 6 (extensive) (table 2). One patient with exclusively lateral lead involvement (V4-6) by pathological Q waves was excluded from this analysis. There was a greater chance that the patients with 
Table 2 The impact of the extent of lead involvement by $Q$ waves on the predictability of myocardial scarring

\begin{tabular}{lllll}
\hline \multicolumn{2}{l}{ Septal $Q$ waves } & & & \multicolumn{2}{l}{ Extensive anterior $Q$ waves } \\
\cline { 1 - 1 } Patient & Scar? & & Patient & Scar? \\
\hline 8 & Yes & & 2 & Yes \\
11 & No & & 3 & Yes \\
17 & Yes & & 5 & Yes \\
18 & Yes & & 6 & Yes \\
27 & No & 10 & Yes \\
33 & Yes & 20 & Yes \\
& & 23 & Yes \\
& & 30 & Yes \\
& & 31 & Yes
\end{tabular}

Patients with pathological Q waves in the chest leads, and the incidence of matched perfusion-metabolism defects (scars) in the anterior wall. The patients were divided into those with septal $\mathrm{Q}$ waves $(\mathrm{V} 1-3)$ and those with extensive anterior $\mathrm{Q}$ waves $(\mathrm{V} 1-4,6)$.

extensive pathological $\mathrm{Q}$ waves had matched perfusion-metabolism defects than the patients with pathological $Q$ waves in the septal leads alone $(100 \%$ v $67 \%)$. However, the difference did not reach statistical significance, the $\mathrm{p}$ value being just over 0.05 .

To investigate the potential impact of posterior wall involvement on the absence of pathological Q waves, we studied the 31 patients with inferior scars. Among these, there were eight with posterior scars, four of whom had pathological $\mathrm{Q}$ waves in the inferior leads (table 3). When the cohort is considered, there were 44 scarred regions not associated with pathological Q waves. The absence of pathological Q waves in these regions with myocardial scarring cannot be ascribed to the posterior location of the scars except in four cases.

The mean area of the matched defects associated with pathological Q waves on the ECG was $20 \%$ of the left ventricular surface. This was not significantly different from the mean area of matched defects associated with electrocardiographic non-Q wave regions (18\%).

R AFTER Q AS A MARKER FOR HIBERNATING MYOCARDIUM

To demonstrate any relationship between the presence of hibernating myocardium and the maintenance of $\mathrm{R}$ wave after pathological $\mathrm{Q}$ wave, we concentrated on the 39 regions with pathological $\mathrm{Q}$ waves. These were divided

Table 3 The incidence of posterior location of the inferior scars and its relation to pathological $Q$ waves among the 31 patients with inferior wall scars, divided according to the presence of pathological $Q$ waves in the inferior leads

\begin{tabular}{lllll}
\hline \multirow{2}{l}{ Inferior $Q$ wave } & & \multicolumn{2}{l}{ No inferior $Q$ wave } \\
\cline { 1 - 1 } Patient & Posterior scar & & Patient & Posterior scar \\
\hline 1 & No & 3 & Yes \\
4 & No & & 6 & No \\
9 & No & & 8 & No \\
13 & No & & 17 & No \\
14 & No & & 18 & Yes \\
15 & Yes & & 37 & Yes \\
19 & No & 38 & No \\
25 & Yes & & 41 & No \\
26 & No & & 42 & No \\
28 & No & & 45 & No \\
29 & Yes & & 46 & No \\
31 & No & 47 & Yes \\
32 & Yes & 48 & No \\
35 & No & 50 & No \\
36 & No & 53 & No \\
& & 56 & No \\
\hline
\end{tabular}

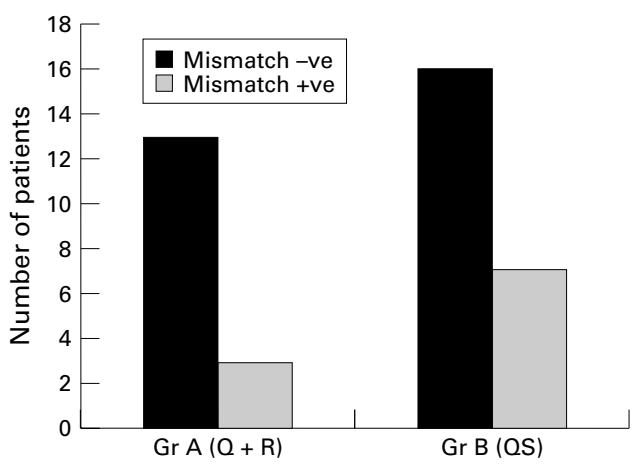

Figure $3 R$ after $Q$ wave as a marker of hibernating myocardium. The bars are representative of two groups of patients with pathological $Q$ waves on their surface ECGs. Group $A$ has $R$ after $Q$ waves, and group $B$ has no $R$ waves after $Q$ waves.

according to the preservation of $\mathrm{R}$ waves after Q waves, into two groups: group A $(n=16)$ comprised the regions with maintained $\mathrm{R}$ waves following the pathological $Q$ waves; group B $(n=23)$ comprised the regions with QS complexes.

In group A, three regions (19\%) showed metabolism-perfusion mismatch defects suggestive of hibernating myocardium. On the other hand, as many as seven regions $(30 \%)$ of the group $\mathrm{B}$ regions were associated with evidence of hibernating myocardium on PET. The difference between the two groups was not statistically significant. Therefore, the presence of $\mathrm{R}$ waves following a pathological $\mathrm{Q}$ wave was not helpful for predicting the presence of hibernating myocardium (fig 3 ).

\section{Discussion}

We investigated the use of the QRS complex on the surface ECG in predicting myocardial viability. This was addressed through two clinical questions.

The first was whether pathological $\mathrm{Q}$ waves are useful markers of myocardial scarring. The second was whether preservation of $R$ waves following pathological $\mathrm{Q}$ waves is a marker for hibernating myocardium.

We found that pathological $\mathrm{Q}$ waves have a high specificity and positive predictive accuracy for myocardial scarring. However, their absence does not exclude the presence of myocardial scarring, given their low sensitivity and low negative predictive accuracy. In addition, the appearance of pathological Q waves is not determined by the size of the matched defect.

In the second question, two groups of regions with $\mathrm{Q}$ waves were compared to test the role of preserved $\mathrm{R}$ waves as a marker of hibernating myocardium. We found that following pathological $\mathrm{Q}$ waves, $\mathrm{R}$ waves are not more likely to be associated with hibernating myocardium than QS complexes. Therefore hibernating myocardium is not more likely to be present if pathological Q waves are followed by $\mathrm{R}$ rather than $\mathrm{S}$ waves.

Detection of viable myocardium is important in the presence of impaired left ventricular contraction, where revascularisation can lead to functional improvement. This can be best predicted using PET. $^{12}$ 
However, viability studies are expensive, hence the need to find ways of selecting patients to undergo these studies, to limit expenditure. The selection process cannot rely on factors such as the frequency of angina, the presence of congestive heart failure, the extent of left ventricular impairment or the presence of serious arrhythmia. These variables do not reliably differentiate between patients with and without residual myocardial viability. ${ }^{6}$

It has been proposed that the preservation of $\mathrm{R}$ waves, following pathological $\mathrm{Q}$ waves, may be a marker for hibernating myocardium, ${ }^{2}$ but this has not been tested against imaging techniques for myocardial viability. As for pathological Q waves, their relation to myocardial viability has been controversial.

Those who believe that pathological $Q$ waves represent myocardial scarring cite evidence of increased myocardial necrosis (increased creatine kinase levels), myocardial impairment, and a worse prognosis in association with $\mathrm{Q}$ wave myocardial infarction. ${ }^{3-5}$

Those who doubt the relation between $Q$ waves and myocardial scarring, however, rely on the presence of myocardial viability in areas corresponding to the electrocardiographic regions with $\mathrm{Q}$ waves, ${ }^{6}$ and the presence of only mild to moderate degree of fibrosis in up to $20 \%$ of biopsies of the akinetic or dyskinetic anterior myocardial segments with $\mathrm{Q}$ waves on the ECG. ${ }^{13}$

One of the problems of investigating the role of the QRS complex in the prediction of myocardial viability is the unreliability of the ECG in localising pathological processes to specific myocardial regions. In our investigation, we avoided the detailed division of the rather complicated septal, anterior, and lateral regions of the left ventricular myocardium. The left ventricular myocardium was therefore divided into two major regions, anterior and inferior. However, we did look at whether extensive pathological $Q$ waves in the chest leads $(\mathrm{V} 1-4,6)$ are more likely to predict scarring than the limited appearance of these waves in the septal leads (V1-3). There was no statistically significant difference in the incidence of scarring between the two subgroups. However, a potential confounding factor is the small number of patients in each subgroup (table 2).

In the setting of posterior myocardial infarction, overt $\mathrm{Q}$ waves may not develop on the classical surface ECG. The patient may have an increased $\mathrm{R}$ to $\mathrm{S}$ ratio anteriorly, and particularly when the infarct related artery is the left circumflex artery these patients may have diminution of the $\mathrm{R}$ wave amplitude in the lateral leads (I, aVL, and V5-6). However, this problem was encountered in four of the 44 regions with scars and no corresponding pathological Q waves.

A third potential confounding factor in the investigation of myocardial viability is the use of ECGs recorded in the acute or subacute phases of myocardial infarction. Q waves may be a temporary electrical expression of myocardial injury during these phases of myocardial infarction rather than a sign of permanent myocardial damage. ${ }^{14}$ Therefore, all ECGs studied were recorded at a minimum of six weeks after myocardial infarction, with the mean time from the index infarct to the ECG recording being 109 weeks (range six weeks to 13 years).

Some of the patients $(22 \%)$ never had a clinically documented myocardial infarct. In these patients, the occurrence of infarction was evident from the demonstration of severe wall motion abnormality on non-invasive or invasive imaging, with or without an occluded infarct related artery on coronary angiography. In these patients, PET was carried out at least six weeks following their presentation, during which they neither experienced symptoms suggestive of myocardial infarction nor developed any new electrocardiographic evidence of infarction.

The present study extends the previous work of Brunken et al. ${ }^{6}$ In a small group of 20 patients, these investigators found that about $40 \%$ of regions with $Q$ waves and severe hypokinesis (or worse function) on wall motion study will show metabolic activity on PET. In our study, a larger cohort of patients was studied, likely to be representative of a typical group of patients who will be considered for myocardial viability studies.

Ragosta et al used rest-redistribution planar thallium- 201 to detect myocardial viability in 21 patients undergoing myocardial revascularisation. ${ }^{15}$ There were 11 patients whose left ventricular contraction did not improve significantly after surgery (and therefore had scarred myocardium). These were significantly more likely to have Q waves on the ECG (91\% of the patients in this group $v 40 \%$ in the remaining group, $\mathrm{p}=0.02$ ).

Their finding suggests that $\mathrm{Q}$ waves are more likely to be associated with scarred than with hibernating myocardium. This is in keeping with our conclusion that $\mathrm{Q}$ waves have high specificity and positive predictive accuracy for myocardial scarring.

In conclusion, the presence of pathological $Q$ waves does not exclude the presence of viable myocardium. However, it indicates a high probability of some scarring in the corresponding myocardium. The reverse is not true, as the negative predictive accuracy of $Q$ waves as markers of scarring is poor. The presence of hibernating myocardium in areas with $\mathrm{Q}$ waves is not reflected by preservation of $R$ waves. Further studies into simple clinical markers for myocardial viability are required.

We are grateful to Mrs F Chilcot, Mr M Dodds, and Miss F Chaloner of the Aberdeen PET Unit for technical assistance. This study was supported, in part, by a grant from the Aberdeen Royal Hospitals NHS Trust Endowment Fund.

1 Sanz G, Castaner A, Betriu A, et al. Determinants of prog nosis in survivors of myocardial infarction. $N$ Engl f Med 1982;306:1065-70.

2 Brookes CI, Hart P, Keogh BE, et al. Angiography and the aetiology of heart failure. Postgrad Med F 1995;71:480-2.

3 Barbagelata A, Califf RM, Sgarbossa EB, et al. Thrombolysis and $\mathrm{Q}$ wave versus non- $\mathrm{Q}$ wave first acute myocardial infarction: a GUSTO-I substudy. $\mathcal{f} \mathrm{Am}$ Coll Cardiol 1997;29:770-7.

4 Isselbacher EM, Siu SC, Weyman AE, et al. Absence of Q waves after thrombolysis predicts more rapid improvement of regional left ventricular dysfunction. Am Heart $f$ 1996;131:649-54.

5 Matetzky S, Barabash GI, Rabinowitz B, et al. Q wave and non-Q wave myocardial infarction after thrombolysis. $7 \mathrm{Am}$ Coll Cardiol 1995;26:1445-51.

6 Brunken R, Tillisch J, Schwaiger M, et al. Regional perfusion, glucose metabolism and wall motion in patients 
with chronic electrocardiographic $\mathrm{Q}$ wave infarctions: evidence for persistence of viable tissue in some infarct regions by positron emission tomography. Circulation 1986;

7 Al-Mohammad A, Mahy IR, Norton MY, et al. Prevalence of hibernating myocardium in patients with severely impaired ischaemic left ventricles. Heart 1998;80:559-64 8 Chan RK, Lee KJ, Calafiore P, et al. Comparison of dobutamine echocardiography and positron emission tomography in patients with chronic ischaemic left ventricular dysfunction. 7 Am Coll Cardiol 1996;27:1601-7.

9 Adams JN, Norton $M$, Trent RJ, et al. Incidence of hibernating myocardium after acute myocardial infarction treated with thrombolysis. Heart 1996;75:442-6.

10 Goris ML, Thomson C, Malone LJ, et al. Modelling the integration of myocardial regional perfusion and function. Nucl Med Commun 1994;15:9-20.

11 Georg Wolpers H, Burchert W, van den Hoff J, et al. Assessment of myocardial viability by use of ${ }^{11} \mathrm{C}$-acetate and posi- tron emission tomography, threshold criteria of reversible dysfunction. Circulation 1997;95:1417-24.

12 Tillisch JH, Brunken R, Marshall R, et al. Reversibility of cardiac wall motion abnormalities predicted by positron tomography. N Engl f Med 1986;314:884-8.

13 Stinson EB, Billingham ME. Correlative study of regional left ventricular histology and contractile function. $\mathrm{Am} \mathcal{F}$ Cardiol 1977;39:378-83.

14 Raitt MH, Maynard C, Wagner GS, et al. Appearance of abnormal Q waves early in the course of acute myocardial infarction: implications for efficacy of thrombolytic therapy. F Am Coll Cardiol 1995;25:1084-8.

15 Ragosta M, Beller GA, Watson DD, et al. Quantitative planar rest-redistribution $201 \mathrm{Tl}$ imaging in detection of myocardial viability and prediction of improvement in left ventricular function after coronary bypass surgery in patients with severely depressed left ventricular function. Circulation 1993;87:1630-41.

\section{Left main coronary artery lesion detected by transoesophageal echocardiography before cardioversion}

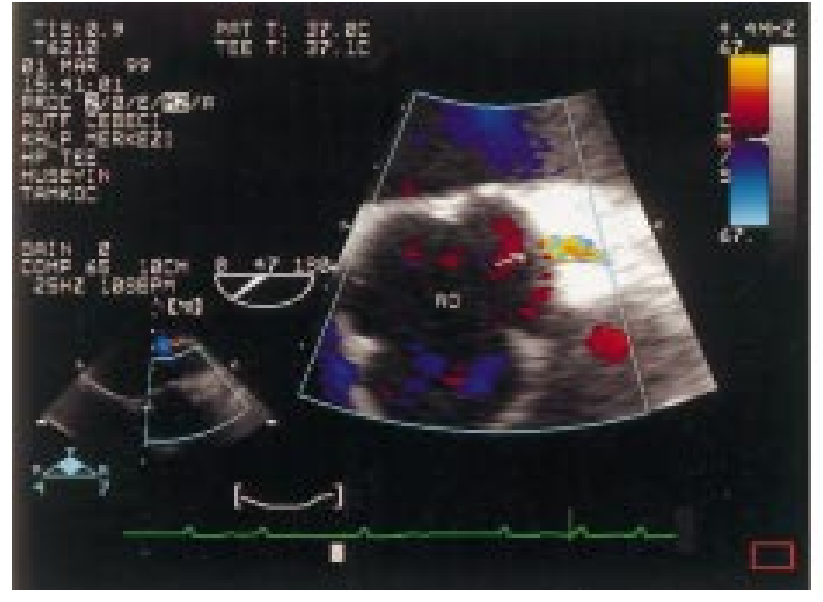

A 60 year old white man with history of hypertensive heart disease had complained of dyspnoea for a month. Newly developed atrial fibrillation caused symptomatic deterioration. Routine precardioversion transoesophageal echocardiography to rule out left atrial thrombus under oral anticoagulation had revealed a mosaic pattern in the left main coronary artery (LMCA) ostium. Colour Doppler imaging was performed to determine whether there was a significant left main coronary artery lesion. Coronary angiography showed ostial stenosis of the left main coronary artery without any other lesion in the left or right coronary artery system. We detected 35\% diameter stenosis

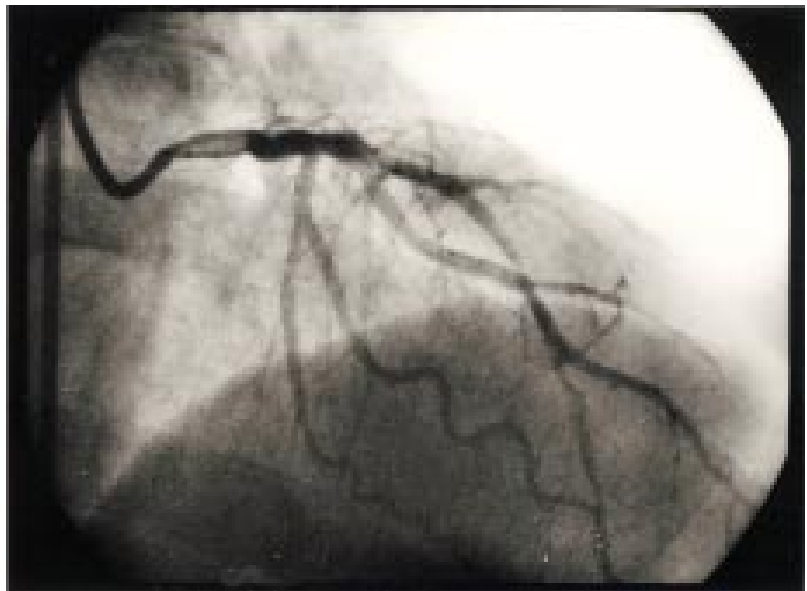

and $58 \%$ area stenosis in the LMCA ostium. Minimal lumen diameter and reference segment diameter were calculated to be $3.13 \mathrm{~mm}$ and $4.83 \mathrm{~mm}$, respectively. Although the lumen diameter $(>3 \mathrm{~mm})$ was sufficient for coronary artery perfusion, intravascular ultrasound imaging was performed, which showed a non-critical eccentric lesion with mixed plaque composition, causing $27 \%$ diameter stenosis and $46.5 \%$ area stenosis. The patient underwent cardioversion safely.

O DOVEN

B BERKALP

D ORAL 